\title{
Unmanned Aerial Vehicle (UAV) Cooperative Mission Planning
}

\author{
Le Yu, Qian Liu \\ Graduate Department, Beijing WuZi University, Beijing, China \\ Email address: \\ yulerunning@163.com (Le Yu), 1767378571@qq.com (Qian Liu)
}

\section{To cite this article:}

Le Yu, Qian Liu. Unmanned Aerial Vehicle (UAV) Cooperative Mission Planning. American Journal of Engineering and Technology Management. Vol. 2, No. 4, 2017, pp. 36-44. doi: 10.11648/j.ajetm.20170204.11

Received: March 27, 2017; Accepted: April 12, 2017; Published: October 24, 2017

\begin{abstract}
Unmanned Aerial Vehicle (UAV) is a kind of new operational platform possessing ability to flight autonomously and perform independently a task, which can not only carry out non-attack tasks,such as military reconnaissance, surveillance and search, but also to carry out tasks to air-to-ground attacking, target bombing and so on. With the rapid development of UAVtechnology, more and more UAV will be applied in the future battlefield. An UAV combat troops have seven UAV bases, which are from P01 to P07. Every base has some FY-1 type UAVs. At the same time, FY-1 UAV can be loaded by two kinds of load, which are S-1 and S-2. Now we need to achieve the aim to detect 10 target groupsfromA01 to A10, which are total 68 goals. And each target group has radar station. Under the above condition, this papermakes the best plan for the UAV combat troops, and uses FY-1 UAV to find best route and scheduling strategy of UAV, which including each UAV drone off base, loading, departure time, track and target reconnaissance. The goal is to ensure minimum time summation in a effective probe range to stay defense radar for UAV. First of all, this paper considers only four UAV bases with FY-1 UAV, so the 68 targets are divided into four regions by K-means algorithm; Then the global shortest path model is established, when the local route is the shortest. The route is drawn according to the route. According to the former route, the general shortest path model is established. It is composed of shortest route distance and the distance from UAV to the corresponding area. And then this paper determine which base the UAV will go to. Finally, the minimum time is calculated as $17.52 \mathrm{~h}$. The eight UAVs are arranged in this process, which are composed of four UAVs withS-1 and fourUAVs withS-2. The UAVs are offered by P01, P03, P05 and P07.
\end{abstract}

Keywords: Multi UAV Cooperative, Task Planning, K-Means Algorithm, Dynamic Time Window

\section{Introduction}

Unmanned Aerial Vehicle (UAV), is a unmanned plane to use wireless remote control equipment and own program control device to control oneself, whichinstalls Autopilot,program control device, etc.Ground control station personnel use transmitting station device to tracking, positioning,remote control, telemetry and digital transmission for UAV [1]. UAV can take off like ordinary plane under the wireless remote control or be used launching cradle to blast offin the military, the UAVs not only are widely used and low cost, but also has the advantages of no casualties risk and great viability [2]. UAV plays a very important role in the modern war, and has broad prospects in the civil field.

Unmanned Aerial Vehicles have great significance to the future air combat.Thereforethe world's major military countries are stepping up the development of UAV.However, the route and operational strategy of UAV are the key factors that affect the operation of UAV directly. The key factor to the successful completion of the combat mission are a reasonable running route and detection of a target.Therefore, this paper,used a specific example to build model and design reasonable flight path and strategy, which provided a new operation scheme for UAV application in military. This is the significance of the paper.

\section{Restatement of the Problem}

UAV combat troops have P01 P07 7 UAV bases. Each base have some FY-1 UAV(The specific coordinates and number of each base can be seen inTable 1. The location diagram can be seen in Figure 1). The main purpose of the FY-1 UAV is the target reconnaissance and target indication. It'scruising speed is $200 \mathrm{~km} / \mathrm{h}$, the longest cruise time is $10 \mathrm{~h}$, 
the cruising altitude is $1500 \mathrm{~m}$;Due to the lack of fuels,UAVs try to reduce the turning, climbing, diving and other maneuvers during the flight. In general, Fuel consumption of maneuvering are $2 \sim 4$ times bigger than cruise. The minimum turning radius is $70 \mathrm{~m}$.

FY-1 UAV can load S-1 and S-2.The load S-1 imaging sensor uses the wide area search mode to image the target, and the imaging bandwidth of the sensor is $2 \mathrm{~km}$; Load S-2 is optical sensor, in order to achieve a certain target recognition accuracy, when the ground target requires no more than $7.5 \mathrm{~km}$ distance from the camera, the camera can be completed immediately.Due to the limitations of various technical conditions, FY-1 UAV can be loaded one of S-1 and $\mathrm{S}-2$.To ensure the effectiveness of the reconnaissance, $\mathrm{S}-1$ and S-2 should be arranged to detect at least once for every target.Investigation interval time of two different loads on the same target is not more than 4 hours. When the two FY-1 UAVsfly in the air, the distance are more than $200 \mathrm{~m}$. Due to the limitations of the base logistics technical support, the two UAVs time interval of take-off and descent on same bases aremore than 3 minutes. UAV needs to return to the original base after the completion of the mission.

FY-1 UAV is now required to complete the reconnaissance targets such as A01 A10 10 target groups. Each group contains the number of ground targets, a radar station for each target group are assigned (The location of the targets and the radar of each target group is shown in Figure 1. The specific coordinates are shown in Table 2). The effective detection range of the radar target group on FY-1are $70 \mathrm{~km}$.But once the reconnaissance UAV enter into radar detection rangeof defending a target group.The radar of ten targets works for alert and search targets and take the corresponding measures, including missiles launched to destroy UAV etc. Therefore, the longer the reconnaissance UAV detained the defense radar detection range, the greater the likelihood of its destruction. To complete the reconnaissance mission of FY-1 UAV to 10 target groups (a total of 68 targets). This paper needs to find best route and scheduling strategy of UAV (including each UAV drone off base, loading, departure time, track and target reconnaissance). The goal is to ensure minimum time summation in a effective probe range to stay defense radar for UAV.

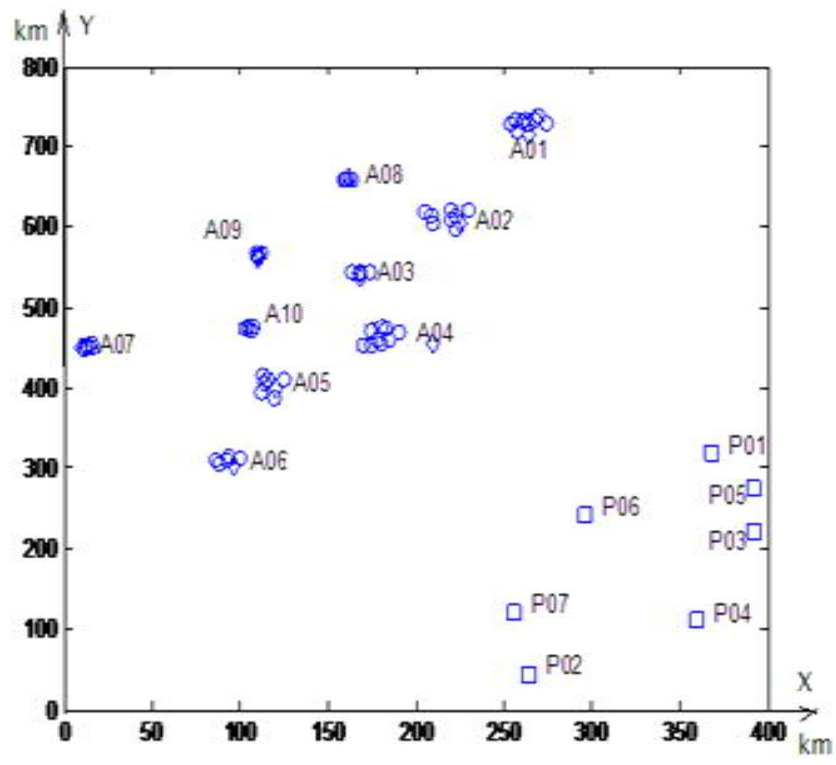

Figure 1. Schematic diagram of target groups and UAV bases.

Table 1. Coordinate and FY-1 attachment quantity of UAV bases.

\begin{tabular}{|c|c|c|c|c|c|c|c|}
\hline Base name & P01 & P02 & P03 & P04 & P05 & P06 & P07 \\
\hline (X, Y)(unit: km) & $(368,319)$ & $(264,44)$ & $(392,220)$ & $(360,110)$ & $(392,275)$ & $(296,242)$ & $(256,121)$ \\
\hline The quantity of FY-1 & 2 & 0 & 2 & 0 & 2 & 0 & 2 \\
\hline
\end{tabular}

Table 2. Target and radar station location.

\begin{tabular}{|c|c|c|c|c|c|}
\hline Pointname & $(\mathrm{X}, \mathrm{Y})$ (unit: km) & Remarks & Point name & $(\mathrm{X}, \mathrm{Y})$ (unit: km) & Remarks \\
\hline Target group A01 & & & Target group A05 & & \\
\hline A0101 & $(264,715)$ & Radarstation & A0501 & $(120,400)$ & Radar station \\
\hline A0102 & $(258,719)$ & & A0502 & $(119,388)$ & \\
\hline A0103 & $(274,728)$ & & A0503 & $(112,394)$ & \\
\hline A0104 & $(264,728)$ & & A0504 & $(125,410)$ & \\
\hline A0105 & $(254,728)$ & & A0505 & $(114,405)$ & \\
\hline A0106 & $(257,733)$ & & A0506 & $(116,410)$ & \\
\hline A0107 & $(260,731)$ & & A0507 & $(113,416)$ & \\
\hline A0108 & $(262,733)$ & & & & \\
\hline A0109 & $(268,733)$ & & Target group A06 & & \\
\hline \multirow[t]{2}{*}{ A0110 } & $(270,739)$ & & A0601 & $(96,304)$ & Radar station \\
\hline & & & A0602 & $(88,305)$ & \\
\hline Target group A02 & & & A0603 & $(100,312)$ & \\
\hline A0201 & $(225,605)$ & Radar station & A0604 & $(93,311)$ & \\
\hline A0202 & $(223,598)$ & & A0605 & $(86,310)$ & \\
\hline
\end{tabular}




\begin{tabular}{|c|c|c|c|c|c|}
\hline Pointname & $(\mathrm{X}, \mathrm{Y})$ (unit: km) & Remarks & Point name & $(X, Y)$ (unit: km) & Remarks \\
\hline A0203 & $(210,605)$ & & A0606 & $(94,315)$ & \\
\hline A0204 & $(220,610)$ & & & & \\
\hline A0205 & $(223,615)$ & & & & \\
\hline A0206 & $(209,615)$ & & Target group A07 & & \\
\hline A0207 & $(230,620)$ & & A0701 & $(10,451)$ & Radar station \\
\hline A0208 & $(220,622)$ & & A0702 & $(11,449)$ & \\
\hline \multirow[t]{2}{*}{ A0209 } & $(205,618)$ & & A0703 & $(13,450)$ & \\
\hline & & & A0704 & $(16,450)$ & \\
\hline Target group A03 & & & A0705 & $(12,453)$ & \\
\hline A0301 & $(168,538)$ & Radar station & A0706 & $(15,455)$ & \\
\hline A0302 & $(168,542)$ & & & & \\
\hline A0303 & $(164,544)$ & & Target group A08 & & \\
\hline A0304 & $(168,545)$ & & A0801 & $(162,660)$ & Radar station \\
\hline \multirow[t]{2}{*}{ A0305 } & $(174,544)$ & & A0802 & $(161,659)$ & \\
\hline & & & A0803 & $(159,659)$ & \\
\hline Target group A04 & & & A0804 & $(160,657)$ & \\
\hline A0401 & $(210,455)$ & Radar station & A0805 & $(164,658)$ & \\
\hline A0402 & $(180,455)$ & & & & \\
\hline A0403 & $(175,452)$ & & Target group A09 & & \\
\hline A0404 & $(170,453)$ & & A0901 & $(110,561)$ & Radar station \\
\hline A0405 & $(185,460)$ & & A0902 & $(110,563)$ & \\
\hline A0406 & $(178,460)$ & & A0903 & $(110,565)$ & \\
\hline A0407 & $(190,470)$ & & A0904 & $(109,567)$ & \\
\hline A0408 & $(183,473)$ & & A0905 & $(112,568)$ & \\
\hline A0409 & $(175,472)$ & & & & \\
\hline \multirow[t]{6}{*}{ A0410 } & $(180,476)$ & & Target group A10 & & \\
\hline & & & A1001 & $(105,473)$ & Radar station \\
\hline & & & A1002 & $(106,471)$ & \\
\hline & & & A 1003 & $(103,473)$ & \\
\hline & & & A1004 & $(107,475)$ & \\
\hline & & & A1005 & 477) & \\
\hline
\end{tabular}

\section{Problem Analysis}

Under the requirements of the problem, this paper solves that FY-1 completes ten target groups task of investigation. Radar of 10 target groups in the range of detection rangesearch and destroy the UAV. Therefore,thegoal is to ensure that the UAV stayed in the radar detection range for a little time to arrange UAVsof the seven bases to complete the task of investigation.In this process, this paper first divides the 10 target groups into the following four areas by using the $\mathrm{K}$-means algorithm in this paper. Then FY-1 UAV on four bases are assigned to each region to perform tasks.At the same time, by understanding the imaging range and bandwidth requirements of the imaging sensor S-1 and the camera range of the optical sensor S-2, the optimal position of the UAV is determined. Next, the shortest path is used to determine the best path in each region [3]. Finally, time limit, detection time interval on the same goal and combat radius ofthe two UAVs in a same baseare considered, which concluded the scheduling strategy and the best way to solve the above problems.

\section{Basic Assumptions}

(1) Assuming that the UAV does not take into account the maneuver, such as turning, climbing and diving;

(2) Assuming that the FY-1 UAV payload can be completed immediately to take the photo reconnaissance mission;

(3) Assuming that the FY-1 UAV is not destroyed in the radar detection range.

\section{Model Development}

\subsection{Building a Model}

Before establishing the model, the necessary symbols are defined as follows:

$\mathrm{i}$-TheIbase $(\mathrm{i}=1 \ldots . .7)$

$\mathrm{j}$-The $\mathrm{j}$ target $(\mathrm{j}=1 \ldots \ldots .68)$

N-Number of UAV

M-Number of tasks to be performed

(Xi,Yi)-Location coordinates of base $\mathrm{i}$

(xj,yj)-Location coordinates of target $\mathrm{j}$ 
dij-The distance between thei base and the $\mathrm{j}$ target

$\mathrm{t}_{i}^{\mathrm{n}}$-Thei base FY-1 UAV take-off time

$\mathbf{t}_{i}^{f}$-The i base FY-1 UAV landing time

Other models involved in the many parameters and symbols are not listed, but in each calculation of the specific explanation below.

In this paper, the target of multi UAV cooperative task allocation is to make the time of multi UAV in the effective detection range in defense radar are as short as possible. That is, $\mathrm{UAV}$ was assigned the task, stayed in the range for a little time.So the objective function is as follows:

$$
\operatorname{MinT}=\sum_{\mathrm{i}=1}^{\mathrm{n}} \sum_{\mathrm{k}=1}^{\mathrm{m}} \mathrm{T}_{\mathrm{k}}
$$

mi is the $\mathrm{i} U A V$ assigned to all the number of targets

UAV cooperative control application scenarios include $\mathrm{N}$ unmanned aerial vehicles and $\mathrm{M}$ tasks to be performed [4]. The task assignment matrix $\mathrm{Sij}$ can be expressed as:

$$
\mathrm{Sij}=\left\{\begin{array}{c}
1, \text { task } \mathrm{j} \text { assigned to UAV } \mathrm{i} \\
0, \text { other }
\end{array}\right.
$$

First of all, it is required that the number of the UAV should not exceed the number of UAVs to be allocated:

$$
\sum_{j=1}^{n} x i \leq N
$$

When multi UAV take missions, the combat radius is also the primary constraint to be considered, that is, the UAV flight distance must be within the scope of its combat radius:

$$
\mathbf{X}_{i, j_{1}} l_{i, j 1}+\sum_{k=2}^{m i} x_{i, j k} l j_{k-1, j k} \leq D i
$$

$\mathrm{Li}, \mathrm{j} 1$ isi UAV to the first target distance;

ijk-1, jkis the $\mathrm{i} U A V$ assigned the distance between the two targets;

$\mathrm{Di}$ is the operational radius of the $\mathrm{i} U \mathrm{UVV}$.

The 10 target groups of the 68 targets are divided into 4 regions need to cluster algorithm, the model is as follows:

$$
\begin{gathered}
\stackrel{\sum_{\text {continous }}^{n} x_{j}}{n}=\frac{x_{x=1}}{\text { Mind }=}=\min \sqrt{\left(y_{i}-c_{k} y_{j}\right)^{\wedge} 2+\left(x_{i}-c_{k} x_{j}\right)^{\wedge} 2} \\
\underset{\text { coutinous }}{\longrightarrow}-\frac{\text { continous }}{\longrightarrow}<\Delta
\end{gathered}
$$

\footnotetext{
$\longrightarrow$ continous $\longrightarrow$ is new cluster center

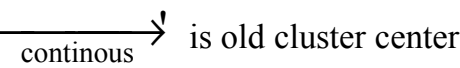

$\Delta$ is the threshold, $\Delta=0.00005$, when the condition is satisfied, stop clustering [5].

Because only the bases P01, P03, P05, P07 has FY-1 type
UAVs, so the four base UAVs is assigned to the four areas, at this time the distance from the base to the region:

$$
\text { Mind }=\min \left\{\sqrt{\left(X i-x_{j}\right)^{\wedge} 2+\left(Y i-y_{j}\right)^{\wedge} 2}\right\}
$$

The distance between the two target center:

$$
\text { Mind }=\min \sqrt{\left(x_{j}-x_{j k}\right)^{\wedge} 2+\left(\mathrm{y}_{j}-y_{j k}\right)^{\wedge} 2}
$$

When $\mathrm{d}>2 \mathrm{R}$, the center distance between the two target shows that:

$$
\begin{aligned}
& \frac{Y_{1}-y}{X i-x} * \frac{y_{i}-y}{x_{j}-x}=-1 \\
& \left(y-y_{j}\right)^{\wedge} 2+\left(x-x_{j}\right)^{\wedge} 2=R^{\wedge} 2
\end{aligned}
$$

When $\mathrm{d} \leqq 2 \mathrm{R}$,

$$
\begin{aligned}
& \left(\mathrm{y}-\mathrm{y}_{\mathrm{j}}\right)^{\wedge} 2+\left(x-x_{j}\right)^{\wedge} 2=R^{\wedge} 2 \\
& \left(y-y_{j k}\right)^{\wedge} 2+\left(x-x_{j k}\right)^{\wedge} 2=R^{\wedge} 2
\end{aligned}
$$

Calculating the shortest distance between each base to four regions, and determining the corresponding relationship between the base and the region:

$$
\operatorname{Min} \mathrm{P}=\min \{\mathrm{P} 1 \mathrm{k} 1+\mathrm{P} 2 \mathrm{k} 2+\mathrm{P} 3 \mathrm{k} 3+\mathrm{P} 4 \mathrm{k} 4\}
$$

Among them, P1ki represents the base P1 to regional ki distance (Ki take $1,2,3,4) \mathrm{k} 1 \neq \mathrm{k} 2 \neq \mathrm{k} 3 \neq \mathrm{k} 4$

The time interval between two different loads for the same target are not more than 4 hours,so,

$$
t_{i b}^{n}-t_{i a}^{n}<4
$$

The two UAVs take-off time interval and the time interval in the same base are not more than 3 minutes,

$$
\begin{aligned}
t_{i b}^{n}-t_{i a}^{n} & >\frac{3}{60} \\
t_{i b}^{f}-t_{i a}^{f} & >\frac{3}{60}
\end{aligned}
$$

The longest cruising time of FY-1 UAV is $10 \mathrm{~h}$,

$$
\begin{aligned}
& t_{i a}^{f}-t_{i a}^{n} \leq 10 \\
& t_{i b}^{f}-t_{i b}^{n} \leq 10
\end{aligned}
$$

\subsection{Resolve the Model}

By the principle of K-means algorithm [6], we used the C language to divided the 68 targets into four regions. Each region contains the number of targets were 14,24,10,20 respectively. The results as shown in the following figure: 


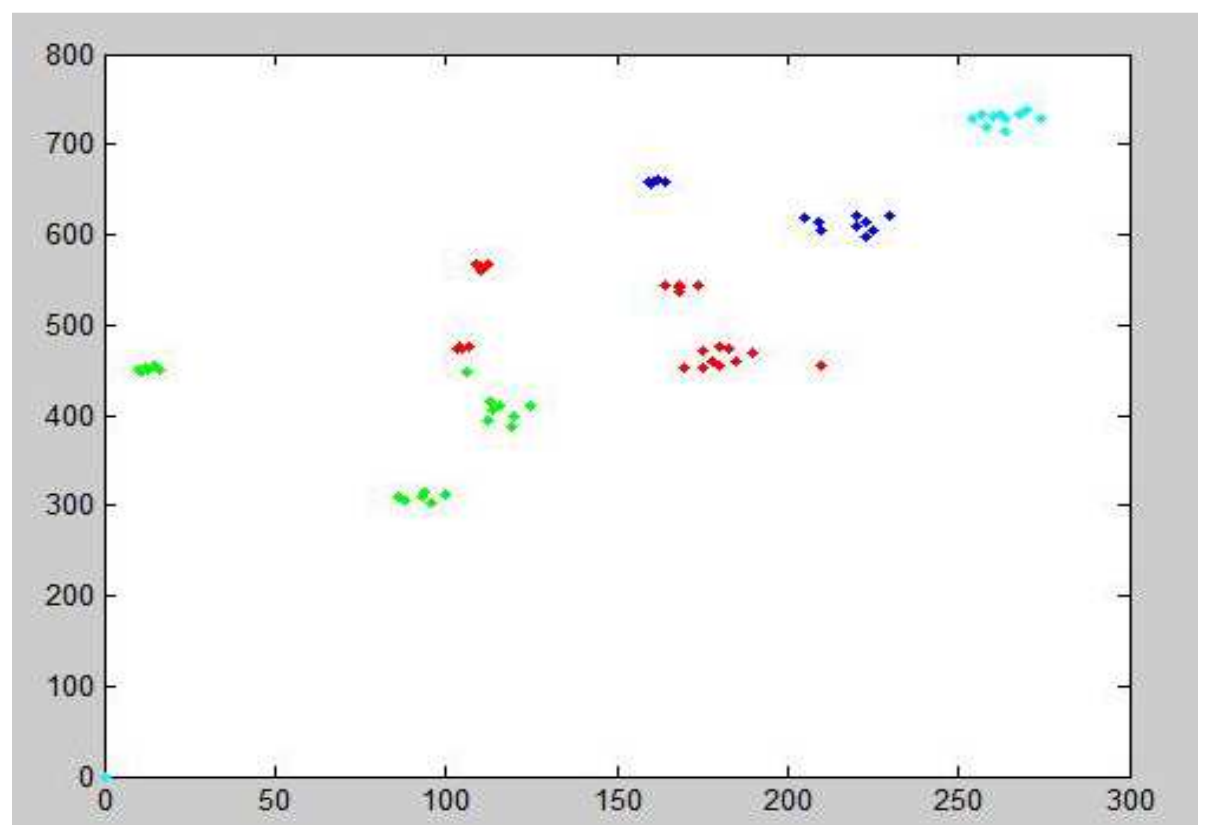

Figure 2. Regions after clustering.

Now there are four FY-I UAV bases with one by one correspondence regions. The shortest distance between them can be assigned as follows:arranging P01 UAV to the blue area of the above, P03 UAV to the red area, P05 UAV to light blue area, P07 UAV to the green area.

By the imaging range of the load S-1 and the camera scope of the S-2, the most appropriate location away from the target is $\mathrm{R} 2=42-1.52$. Thus $\mathrm{UAV}$ tracks are determined. According to the above analysis and data, using MATLAB programming, the circle is painted [7-9]. Coordinate is the center of the circle. UAV reach once the point of circle to finish successfully the task on a point of circle.

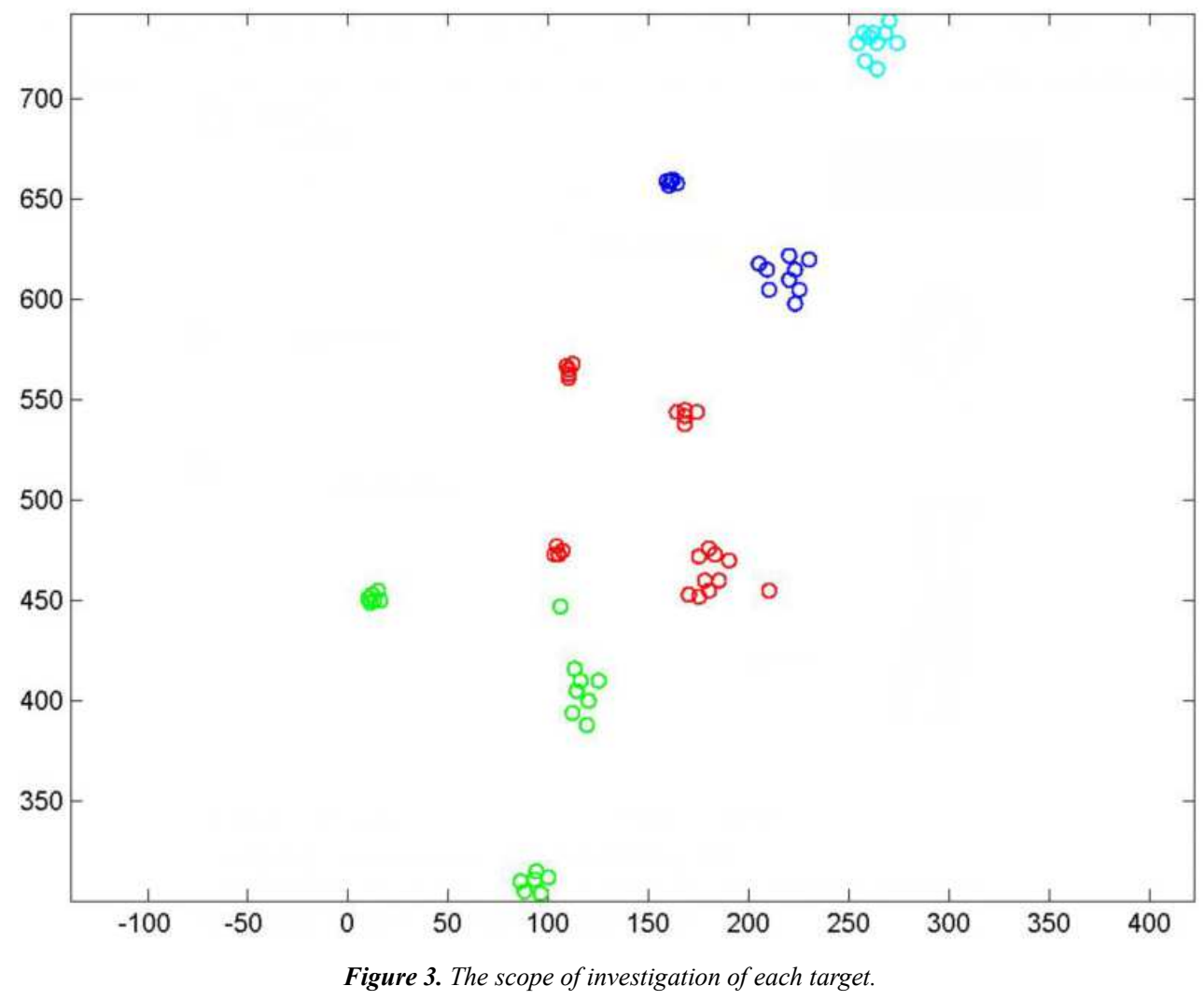

In each area, the shortest path is the path of the UAV when it flights according to the tangent of circle of each target detection range and the intersection location of the two targets
[10]. Thus, the track and the shortest path in the four regions are obtained: 


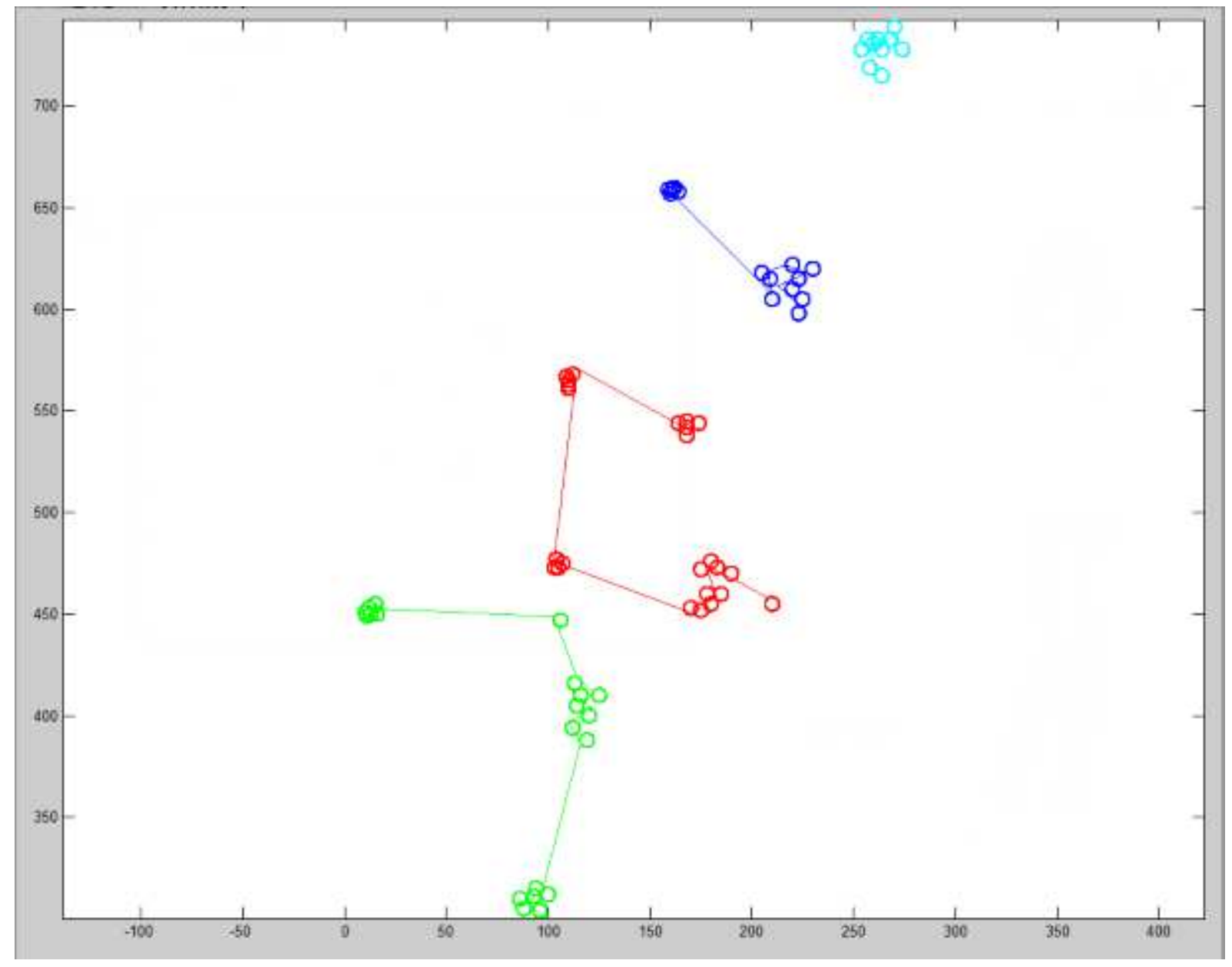

Figure 4. Regional internal track.

For the base P01, arranging for the investigation of the specific area of FY-1 UAV reconnaissance blue area as shown below:

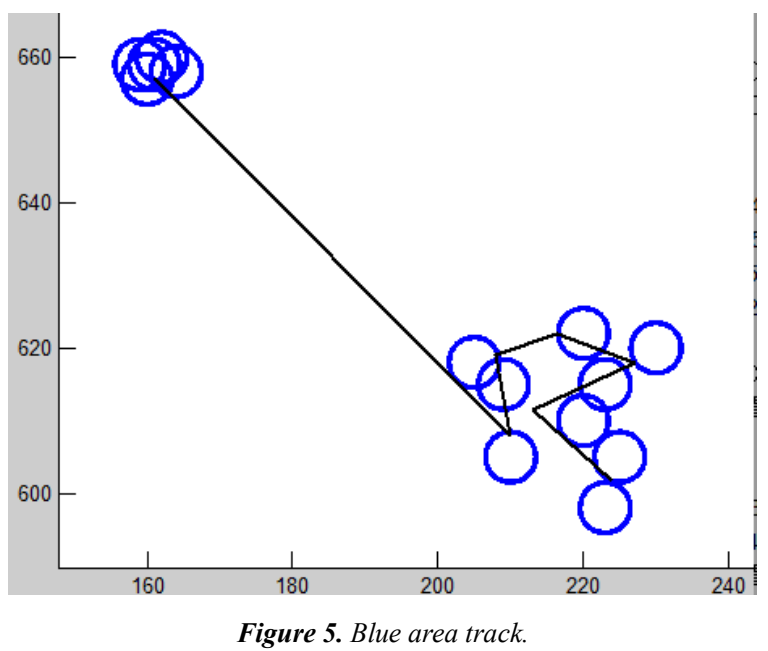

The UAV from the base $(368,319)$ - $(224,601.6)$ $(213,611.5)$ - $(227,618)$ - $(216.5,622)$ - $(208,619)$ - $(210,608)$ $(161,657)$ to search something, and return base after the task. This is the shortest path. And the shortest distance is $846 \mathrm{~km}$.
For the base P03, arranging FY-1 UAVto detect the red area. The specific track as shown below:

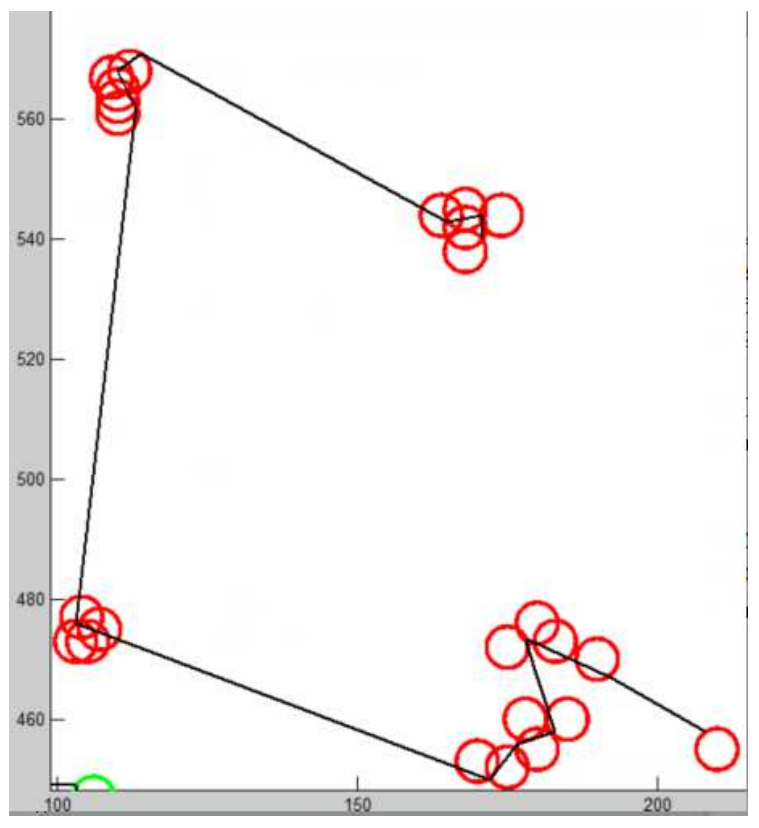

Figure 6. Red area track. 
The UAV from the base $(392,220)-(208,458)-(192,467)$ $(179,473)-(178,473)-(183,458)-(177,56)-(176,455)-$ $(172,450)-(103,476)-(113,562)-(113,564)-(112,568)-$ $(110,571)-(114,571),(165,543),(171,544),(171,540)$ to search something, andreturn base after the task. This is the shortest path.And the shortest distance is $997.2 \mathrm{~km}$.

For the base P05,arranging FY-1 UAV to detect the blue area. The specific track as shown below:

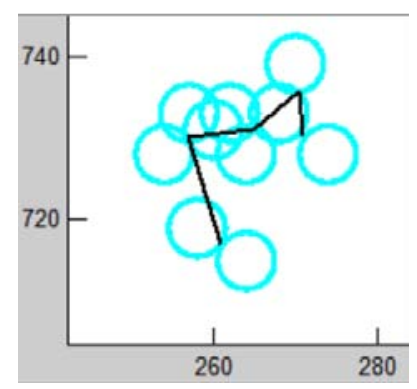

Figure 7. Light blue area track.

The UAV from the base $(392,275)-(261,717)$ - $(257,730)-(265,731)-(270.5,735.6)-(271,730)$ to search something, and return base after the task. This is the shortest path. And the shortest distance is $967.8 \mathrm{~km}$.

For the base P07, arranging FY-1 UAV to detect the green area. The specific track as shown below:

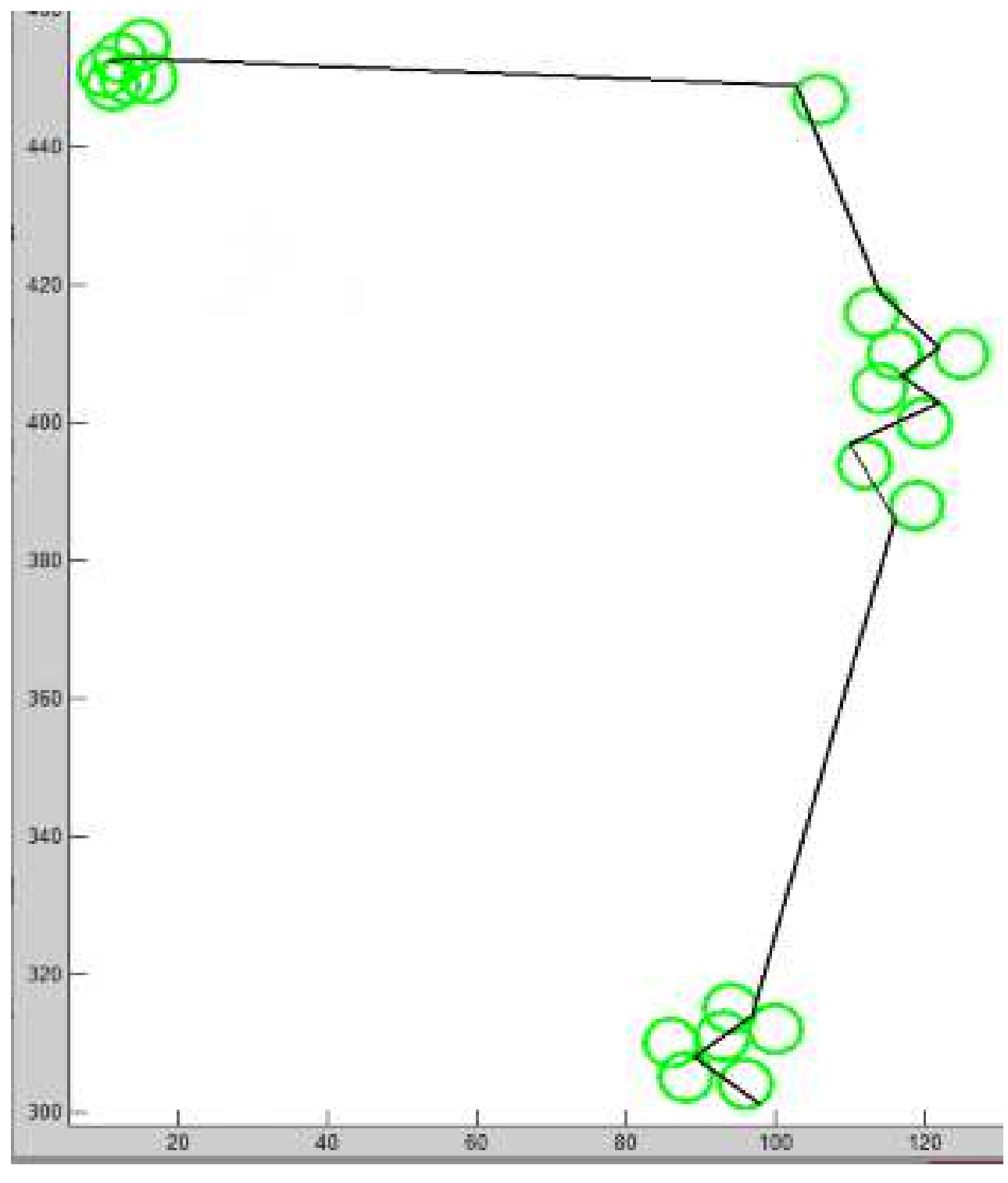

Figure 8. Green area track.

The UAV from the base $(256,121)-(98,301)-(89,308)-$ $(97,314)-(116,386)-(110,397)-(122,403)-(117,407)-$ $(122,411)-(114,419)-(103,449)-(14,453)-(10.5,452.5)$ to search something, and return base after the task. This is the shortest path. And the shortest distance is $924 \mathrm{~km}$.
Because there are 10 radar detectors in 68 goals. The detection distance is $70 \mathrm{~km}$. Detection scope can be detected by the radar. The scope can be seen by the following figure. (Yellow part of the map is the base position): 


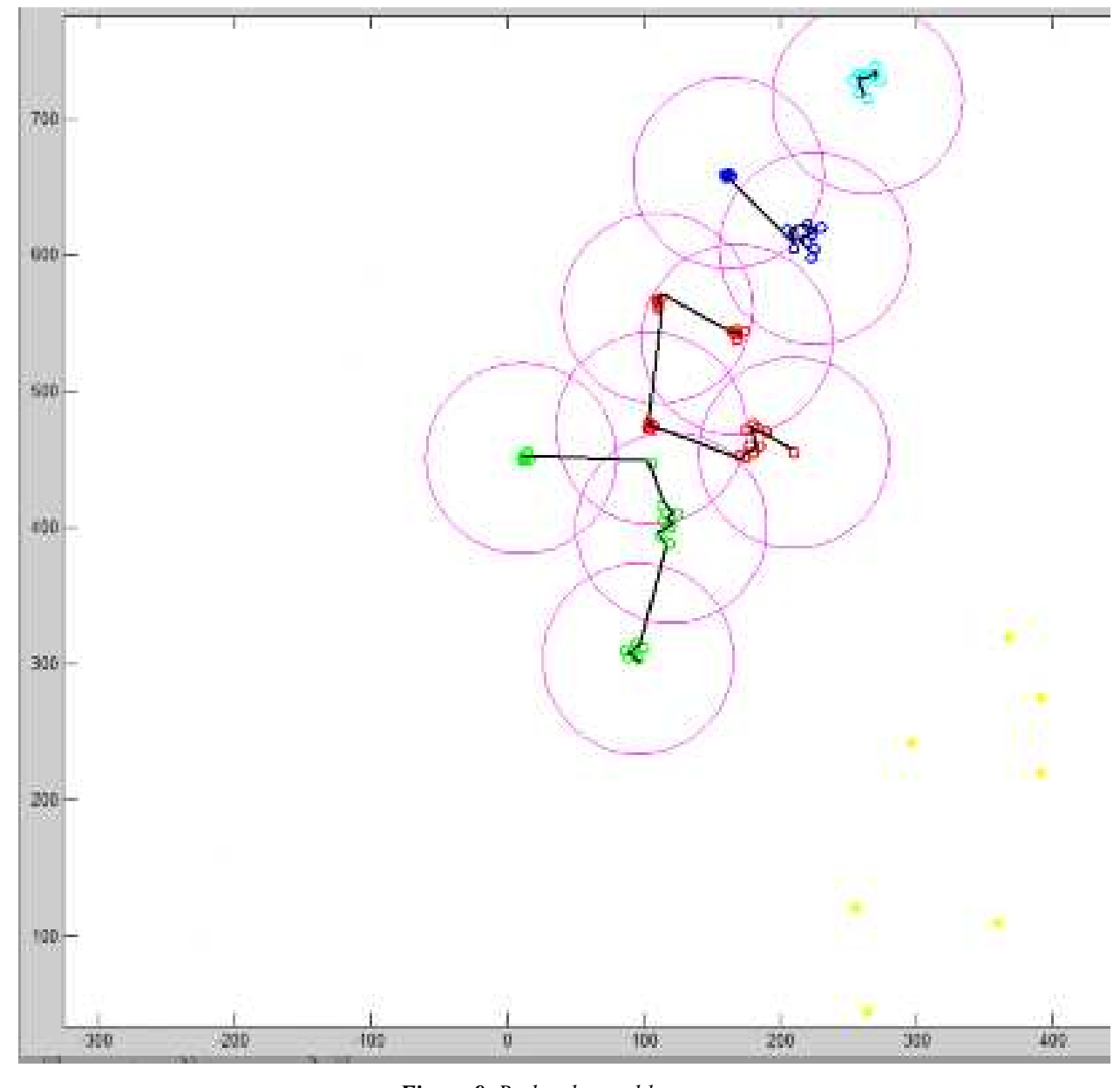

Figure 9. Radar detectable range.

It can be concluded that the distance from the base to each region and the UAV's distance from the radar detection range are as follows [11]:

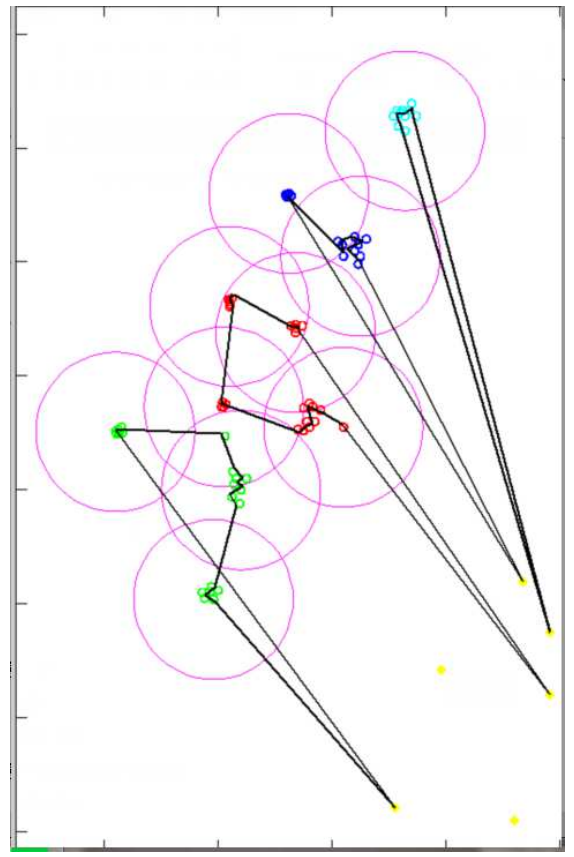

Figure 10. The distance of UAV in radar range.
Because the longest cruise time of type FY-1 is $10 \mathrm{~h}$. The flight speed is $200 \mathrm{~km} / \mathrm{h}$. so the maximum flight distance of the FY-1 UAV is $2000 \mathrm{~km}$. At the same time, from the above data and analysis, the distance traveled by the base to each area is less than the maximum flight distance. That is, each region only sent a S-1 carrying UAVs and a carrier carrying S-2 UAV complete only the task from the base.

The chart can be calculated.The total distance of radar detection range in each regionwere $402.73 \mathrm{~km}, 539.62 \mathrm{~km}$, $235.48 \mathrm{~km}, 574.2 \mathrm{~km}$. So the minimum time summation in the range of detection is $17.52 \mathrm{~h}$.

\section{Results and Analysis}

To sum up, to successfully complete the task of investigation and time summation UAV stayed in the range of detection are little [12]. This problem needs 8 FY-1 UAVs, which are provided 2 respectivelyby the base P01,P03, P05, P07.

The first FY-1 carries S-1, the second FY-1 carries S-2. For the same base, after the first UAV taked off for $3 \mathrm{~min}$,

The second UAV carrying S-2 taked off.This ensures that each UAV can meet all the time constraints. The two

UAVsdetection target of base P01 are A0201, A0202, A0203, A0205, A0206, A0207, A0208,A0204, A0209, A0801, 
A0802, A0803,A0804,A0805; The two UAVsdetection goals of base P03 are A0301,A0302, A0303, A0304, A0305, A0401, A0402,A0403, A0404, A0901, A0405, A0902, A0406, A0903, A0407,A0904, A0408, A0905, A0409, A0410, A1001, A1003, A1004, A1005; The two UAVsdetection goals of base P05 are A0101,A0102, A0103, A0104, A0105, A0106, A0107, A0108, A0109,A0110; The two UAVsdetection goals of base P05are $\mathrm{A} 0501, \mathrm{~A} 0502, \mathrm{~A} 0503, \mathrm{~A} 0504, \mathrm{~A} 0505, \mathrm{~A} 0506, \mathrm{~A} 0507, \mathrm{~A} 0602$, A0603,A0604, A0605, A0606, A0701, A0702, A0703, A0704, $\mathrm{A} 0705, \mathrm{~A} 0706, \mathrm{~A} 1002, \mathrm{~A} 0601$. At present, the minimum time is $17.52 \mathrm{~h}$.

\section{Evaluation and Improvement of the Model}

\subsection{Strengths of the Model}

(1) In the model design, this paper considers the shortest time of flight of UAV, UAV total flight time, multi UAV best route and investigation target coverage degree. This paper consider the number of the UAV, UAV combat radius, the number of combat tasks, task load, high degree of base location, the position of the target audience to build model.It has the feasibility and applicability of the high.

(2) Planning missions in some typical conditions, combining with actual situation, the factors considered comprehensively. The model is reasonable, the algorithm is complete. And the algorithm result canreflect the specific impact of distance, task time, target value for task allocation.

\subsection{Weaknesses of the Model}

Because the variables involved in this paper are too many, the situation is more complex, the main problem is to extract the main analysis of the thinking, weakening the impact of certain variables, so the accuracy of the results are decline; At the same time, the algorithm optimization is simple, the details of the modification is not perfect, which did not achieve the desired results.

\subsection{Improvement of the Model}

(1) Multi UAV cooperative operation requires that each UAV's flight route is the best. In this paper, this papercan not obtain the optimal multi route for the cooperative reconnaissance mission, and also need to modify the model and the algorithm to meet the requirements of multi UAV cooperative mission;

(2) In the scenario assumptions and model assumptions, the assumptions and indicators are still too simple to implement some complex scenarios. So we need more complex conditions and tasks to improve.

\section{References}

[1] D. C. L. Kristen Boon, "Warrant Requirement and Suspicion less Drone Searches," in The Domestic Use of Unmanned Aerial Vehicles, USA, Oxford University Press, 2014, p. 228.

[2] Chen Y B, Luo G C, Mei Y S, et al. UAV path planning using artificial potential field method updated by optimal control theory[J]. International Journal of Systems Science, 2014:1-14.

[3] Ye Yuanyuan. Multi UCAV cooperative task planing method. Doctoral dissertation research. National defense science and Technology University Changsha: National University of Defense Technology, 2015.

[4] Xia Jie. Real time flight path planning to meet the requirements of battlefield [J] Journal of Beihang University, 2014,30 (2): 95-99.

[5] Yang Zun. A monitoring route planning method for unmanned aerial vehicles [J]. tactical missile technology, 2011, (4): 63-67.

[6] Hu Zhonghua. Research and development of UAV mission planning system [J] aerospace electronic warfare, 2009,25 (4): 49-51.

[7] Gong Mao Guo, Wang Shuang, Ma Meng, et al. Two stage clustering algorithm for complex distributed data [J]. Journal of software, 2011,22 (11): 2760-2772.

[8] Fu Xiaowei. UCAV path planning algorithm. Research doctorate dissertation. Xi'an: Northwestern Polytechnical University, 2007.

[9] Roberge V, Tarbouchi M, Labonte G. Comparison of Parallel Genetic Algorithm and Particle Swarm Optimization for Real-Time UAVPath Planning [J]. IEEE Transactions on Industrial Informatics, 2013, 9(1):132-141.

[10] S. Al-Hasan, G. Vachtsevanos. Intelligent Route Planning for Fast Autonomous Vehicles Operatingina Large natural Terrain [J]. Robotics and Autonomous Systems, 2014, 40:1-24.

[11] Changwen Zheng, Lei Li, Fanjiang Xu. Evolutionary Route Planner for Unmanned Air Vehicles [J]. IEEE Transactions on Robotics, 2015, 21(4): 609-620.

[12] Sonia Waharte, Niki Trigoni, Supporting Search and Rescure Operations with UAVs [J]. 2010 International Conference on Emerging Security Technologies, 2010, 2:142-147. 